\title{
QUASI-ALMOST LACUNARY STATISTICAL CONVERGENCE OF SEQUENCES OF SETS
}

\section{ESRA GÜLLE*, UĞUR ULUSU}

Department of Mathematics, Faculty of Science and Literature, Afyon Kocatepe University, 03200, Afyonkarahisar, Turkey

*Corresponding author: egulle@aku.edu.tr

\begin{abstract}
In this study, we defined concepts of Wijsman quasi-almost lacunary convergence, Wijsman quasi-strongly almost lacunary convergence and Wijsman quasi $q$-strongly almost lacunary convergence. Also we give the concept of Wijsman quasi-almost lacunary statistically convergence. Then, we study relationships among these concepts. Furthermore, we investigate relationship between these concepts and some convergences types given earlier for sequences of sets, too.
\end{abstract}

\section{INTRODUCTION AND BACKGROUNDS}

The concept of statistical convergence was first introduced by Fast [10]. Also this concept was studied by Fridy [12], Šalát [17] and many others.

A sequence $x=\left(x_{k}\right)$ is statistically convergent to the number $L$ if for every $\varepsilon>0$,

$$
\lim _{n \rightarrow \infty} \frac{1}{n}\left|\left\{k \leq n:\left|x_{k}-L\right| \geq \varepsilon\right\}\right|=0
$$

where the vertical bars indicate the number of elements in the enclosed set.

Freedman et al. [1] established the connection between the strongly Cesàro summable sequences space $\left|\sigma_{1}\right|$ and the strongly lacunary summable sequences space $N_{\theta}$.

Received 2017-10-26; accepted 2017-12-20; published 2018-03-07.

2010 Mathematics Subject Classification. 40A05, 40A35.

Key words and phrases. almost convergence; quasi-almost convergence; quasi-almost statistical convergence; lacunary sequence; sequences of sets; Wijsman convergence.

(C) 2018 Authors retain the copyrights of their papers, and all open access articles are distributed under the terms of the Creative Commons Attribution License. 
By a lacunary sequence we mean an increasing integer sequence $\theta=\left\{k_{r}\right\}$ such that $k_{0}=0$ and $h_{r}=$ $k_{r}-k_{r-1} \rightarrow \infty$ as $r \rightarrow \infty$. Throughout this study the intervals determined by $\theta$ will be denoted by $I_{r}=\left(k_{r-1}, k_{r}\right]$ and ratio $\frac{k_{r}}{k_{r-1}}$ will be abbreviated by $q_{r}$.

The concept of lacunary statistical convergence was introduced by Fridy and Orhan [13].

Let $\theta=\left\{k_{r}\right\}$ be a lacunary sequence. A sequence $x=\left(x_{k}\right)$ is lacunary statistically convergent to $L$ if for every $\varepsilon>0$,

$$
\lim _{r \rightarrow \infty} \frac{1}{h_{r}}\left|\left\{k \in I_{r}:\left|x_{k}-L\right| \geq \varepsilon\right\}\right|=0 .
$$

The idea of almost convergence was introduced by Lorentz [9]. Maddox [11] and (independently) Freedman [1] gave the concept of strong almost convergence. Similar concepts can be seen in [2].

Let $X$ be any non-empty set and $\mathbb{N}$ be the set of natural numbers. The function

$$
f: \mathbb{N} \rightarrow P(X)
$$

is defined by $f(k)=A_{k} \in P(X)$ for each $k \in \mathbb{N}$, where $P(X)$ is power set of $X$. The sequence $\left\{A_{k}\right\}=$ $\left(A_{1}, A_{2}, \ldots\right)$, which is the range's elements of $f$, is said to be sequences of sets.

Let $(X, \rho)$ be a metric space. For any point $x \in X$ and any non-empty subset $A$ of $X$, we define the distance from $x$ to $A$ by

$$
d(x, A)=\inf _{a \in A} \rho(x, a) .
$$

Throughout the paper we take $(X, \rho)$ as a metric space and $A, A_{k}$ as any non-empty closed subsets of $X$.

There are different convergence notions for sequence of sets. One of them handled in this paper is the concept of Wijsman convergence (see, $[6-8,14-16])$.

A sequence $\left\{A_{k}\right\}$ is said to be Wijsman convergent to $A$ if for each $x \in X$

$$
\lim _{k \rightarrow \infty} d\left(x, A_{k}\right)=d(x, A)
$$

and denoted by $A_{k} \stackrel{W}{\rightarrow} A$ or $W-\lim A_{k}=A$.

A sequence $\left\{A_{k}\right\}$ is said to be bounded if for each $x \in X$

$$
\sup _{k}\left\{d\left(x, A_{k}\right)\right\}<\infty .
$$

The set of all bounded sequences of sets is denoted by $L_{\infty}$.

The concepts of Wijsman statistical convergence was introduced by Nuray and Rhoades [6].

A sequence $\left\{A_{k}\right\}$ is Wijsman statistically convergent to $A$ if for each $x \in X$ and every $\varepsilon>0$

$$
\lim _{n \rightarrow \infty} \frac{1}{n}\left|\left\{k \leq n:\left|d\left(x, A_{k}\right)-d(x, A)\right| \geq \varepsilon\right\}\right|=0
$$

and it is denoted by $s t-\lim _{W} A_{k}=A$.

The concepts of Wijsman lacunary summability $\left(\left(W N_{\theta}\right),[W N]_{\theta},[W N]_{\theta}^{p}\right)$ and concept of Wijsman lacunary statistical convergence $\left(\left(W S_{\theta}\right)\right)$ were introduced by Ulusu and Nuray [20,21]. 
Let $\theta=\left\{k_{r}\right\}$ be a lacunary sequence. A sequence $\left\{A_{k}\right\}$ is Wijsman lacunary summable to $A$ if for each $x \in X$,

$$
\lim _{r \rightarrow \infty} \frac{1}{h_{r}} \sum_{k \in I_{r}} d\left(x, A_{k}\right)=d(x, A)
$$

and it is denoted by $A_{k} \stackrel{\left(W N_{\theta}\right)}{\longrightarrow} A$.

Let $\theta=\left\{k_{r}\right\}$ be a lacunary sequence. A sequence $\left\{A_{k}\right\}$ is Wijsman strongly lacunary summable to $A$ if for each $x \in X$,

$$
\lim _{r \rightarrow \infty} \frac{1}{h_{r}} \sum_{k \in I_{r}}\left|d\left(x, A_{k}\right)-d(x, A)\right|=0
$$

and it is denoted by $A_{k} \stackrel{[W N]_{\theta}}{\longrightarrow} A$.

Let $\theta=\left\{k_{r}\right\}$ be a lacunary sequence. A sequence $\left\{A_{k}\right\}$ is Wijsman p-strongly lacunary summable to $A$ if for each $x \in X$ and $0<p<\infty$,

$$
\lim _{r \rightarrow \infty} \frac{1}{h_{r}} \sum_{k \in I_{r}}\left|d\left(x, A_{k}\right)-d(x, A)\right|^{p}=0
$$

and it is denoted by $A_{k} \stackrel{[W N]_{\theta}^{p}}{\longrightarrow} A$.

A sequence $\left\{A_{k}\right\}$ is Wijsman lacunary statistically convergent to $A$ if for every $\varepsilon>0$ and each $x \in X$,

$$
\lim _{r \rightarrow \infty} \frac{1}{h_{r}}\left|\left\{k \in I_{r}:\left|d\left(x, A_{k}\right)-d(x, A)\right| \geq \varepsilon\right\}\right|=0
$$

and it is denoted by $A_{k} \stackrel{\left(W S_{\theta}\right)}{\longrightarrow} A$.

Also the concepts of Wijsman almost lacunary convergence and Wijsman almost lacunary statistical convergence were introduced by Ulusu $[18,19]$, too.

Let $\theta=\left\{k_{r}\right\}$ be a lacunary sequence. A sequence $\left\{A_{k}\right\}$ is Wijsman almost lacunary convergent to $A$ if for each $x \in X$,

$$
\lim _{r \rightarrow \infty} \frac{1}{h_{r}} \sum_{k \in I_{r}} d\left(x, A_{k+i}\right)=d(x, A)
$$

uniformly in $i=0,1,2, \ldots$

Let $\theta=\left\{k_{r}\right\}$ be a lacunary sequence. A sequence $\left\{A_{k}\right\}$ is Wijsman strongly almost lacunary convergent to $A$ if for each $x \in X$,

$$
\lim _{r \rightarrow \infty} \frac{1}{h_{r}} \sum_{k \in I_{r}}\left|d\left(x, A_{k+i}\right)-d(x, A)\right|=0
$$

uniformly in $i$.

Let $\theta=\left\{k_{r}\right\}$ be a lacunary sequence. A sequence $\left\{A_{k}\right\}$ is Wijsman strongly p-almost lacunary convergent to $A$ if for each $x \in X$ and $0<p<\infty$,

$$
\lim _{r \rightarrow \infty} \frac{1}{h_{r}} \sum_{k \in I_{r}}\left|d\left(x, A_{k+i}\right)-d(x, A)\right|^{p}=0
$$

uniformly in $i$. 
A sequence $\left\{A_{k}\right\}$ is Wijsman almost lacunary statistically convergent to $A$ if for every $\varepsilon>0$ and each $x \in X$,

$$
\lim _{r \rightarrow \infty} \frac{1}{h_{r}}\left|\left\{k \in I_{r}:\left|d\left(x, A_{k+i}\right)-d(x, A)\right| \geq \varepsilon\right\}\right|=0
$$

uniformly in $i$.

The idea of quasi-almost convergence in a normed space was introduced by Hajduković [3]. Then, Nuray [5] studied concepts of quasi-invariant convergence and quasi-invariant statistical convergence in a normed space.

The concepts of Wijsman quasi-strongly almost convergence and Wijsman quasi-almost statistically convergence were studied by Gülle and Ulusu [4].

A sequence $\left\{A_{k}\right\} \in L_{\infty}$ is Wijsman quasi-strongly almost convergent to $A$ if for each $x \in X$,

$$
\frac{1}{p} \sum_{k=n p}^{n p+p-1}\left|d_{x}\left(A_{k}\right)-d_{x}(A)\right| \rightarrow 0 \quad(\text { as } p \rightarrow \infty)
$$

uniformly in $n$ and it is denoted by $A_{k} \stackrel{[W Q F]}{\longrightarrow} A$.

A sequence $\left\{A_{k}\right\}$ is Wijsman quasi-almost statistically convergent to $A$ if for each $x \in X$ and every $\varepsilon>0$

$$
\lim _{p \rightarrow \infty} \frac{1}{p}\left|\left\{k \leq p:\left|d_{x}\left(A_{k+n p}\right)-d_{x}(A)\right| \geq \varepsilon\right\}\right|=0
$$

uniformly in $n$ and it is denoted by $A_{k} \stackrel{W Q S}{\longrightarrow} A$.

The set of all Wijsman quasi-almost statistically convergence sequences will be denoted by $\{W Q S\}$.

\section{MAIN RESULTS}

In this section, we defined concepts of Wijsman quasi-almost lacunary convergence, Wijsman quasistrongly almost lacunary convergence and Wijsman quasi $q$-strongly almost lacunary convergence. Also we give the concept of Wijsman quasi-almost lacunary statistically convergence. Then, we study relationships among these concepts. Furthermore, we investigate relationship between these concepts and some convergences types given earlier for sequences of sets, too.

Definition 2.1. Let $\theta=\left\{k_{r}\right\}$ be a lacunary sequence. A sequence $\left\{A_{k}\right\} \in L_{\infty}$ is Wijsman quasi-almost lacunary convergent to $A$ if for each $x \in X$,

$$
\left|\frac{1}{h_{r}} \sum_{k \in I_{r}} d_{x}\left(A_{k+n r}\right)-d_{x}(A)\right| \longrightarrow 0 \quad(\text { as } r \rightarrow \infty),
$$

uniformly in $n=0,1,2, \ldots$ where $d_{x}\left(A_{k+n r}\right)=d\left(x, A_{k+n r}\right)$ and $d_{x}(A)=d(x, A)$. In this case, we will write $(W Q F)_{\theta}-\lim A_{k}=A$ or $A_{k} \stackrel{(W Q F)_{\theta}}{\longrightarrow} A$. 
Example 2.1. Let we define a sequence $\left\{A_{k}\right\}$ as follows:

$$
A_{k}:= \begin{cases}\left\{x \in \mathbb{R}: 2 \leq x \leq k_{r}-k_{r-1}\right\} & , \text { if } k \geq 2 \text { and } k \text { is square integer }, \\ \{1\} & , \text { otherwise. }\end{cases}
$$

This sequence is not Wijsman lacunary summable. But, since for each $x \in X$

$$
\lim _{r \rightarrow \infty}\left|\frac{1}{h_{r}} \sum_{k \in I_{r}} d_{x}\left(A_{k+n r}\right)-d_{x}(\{1\})\right|=0
$$

uniformly $n$, this sequence is Wijsman quasi-almost lacunary convergent to the set $A=\{1\}$.

Theorem 2.1. If a sequence $\left\{A_{k}\right\} \in L_{\infty}$ is Wijsman almost lacunary convergent to A, then $\left\{A_{k}\right\}$ is Wijsman quasi-almost lacunary convergent to A.

Proof. Suppose that the sequence $\left\{A_{k}\right\}$ is Wijsman almost lacunary convergent to $A$. Then, for each $x \in X$ and every $\varepsilon>0$ there exists an integer $r_{0}>0$ such that for all $r>r_{0}$

$$
\left|\frac{1}{h_{r}} \sum_{k \in I_{r}} d_{x}\left(A_{k+i}\right)-d_{x}(A)\right|<\varepsilon,
$$

uniformly in $i$. If $i$ is taken as $i=n r$, then we have

$$
\left|\frac{1}{h_{r}} \sum_{k \in I_{r}} d_{x}\left(A_{k+n r}\right)-d_{x}(A)\right|<\varepsilon,
$$

uniformly in $n$. Since $\varepsilon>0$ is an arbitrary, the limit is taken for $r \rightarrow \infty$ we can write

$$
\left|\frac{1}{h_{r}} \sum_{k \in I_{r}} d_{x}\left(A_{k+n r}\right)-d_{x}(A)\right| \longrightarrow 0
$$

uniformly in $n$. That is, the sequence $\left\{A_{k}\right\}$ is Wijsman quasi-almost lacunary convergent to $A$.

Theorem 2.2. If a sequence $\left\{A_{k}\right\} \in L_{\infty}$ is Wijsman quasi-almost lacunary convergent to $A$, then $\left\{A_{k}\right\}$ is Wijsman lacunary summable to A.

Proof. Assume that the sequence $\left\{A_{k}\right\} \in L_{\infty}$ is Wijsman quasi-almost lacunary convergent to $A$. Then, Equation (2.1) is true which for $n=0$ implies for every $\varepsilon>0$ and each $x \in X$,

$$
\left|\frac{1}{h_{r}} \sum_{k \in I_{r}} d_{x}\left(A_{k}\right)-d_{x}(A)\right| \longrightarrow 0 \quad(\text { as } r \rightarrow \infty) ;
$$

so, $\left\{A_{k}\right\}$ is Wijsman lacunary summable to $A$.

Definition 2.2. Let $\theta=\left\{k_{r}\right\}$ be a lacunary sequence. A sequence $\left\{A_{k}\right\}$ is Wijsman quasi-almost lacunary statistically convergent to $A$ if for each $x \in X$ and every $\varepsilon>0$

$$
\lim _{r \rightarrow \infty} \frac{1}{h_{r}}\left|\left\{k \in I_{r}:\left|d_{x}\left(A_{k+n r}\right)-d_{x}(A)\right| \geq \varepsilon\right\}\right|=0,
$$


uniformly in $n$. In this case, we will write $(W Q S)_{\theta}-\lim A_{k}=A$ or $A_{k} \stackrel{(W Q S)_{\theta}}{\longrightarrow} A$.

The set of all Wijsman quasi-almost lacunary statistically convergence sequences will be denoted by $\left\{W Q S_{\theta}\right\}:$

$$
\left\{W Q S_{\theta}\right\}=\left\{\left\{A_{k}\right\}: \lim _{r \rightarrow \infty} \frac{1}{h_{r}}\left|\left\{k \in I_{r}:\left|d_{x}\left(A_{k+n r}\right)-d_{x}(A)\right| \geq \varepsilon\right\}\right|=0\right\} .
$$

Theorem 2.3. If a sequence $\left\{A_{k}\right\}$ is Wijsman almost lacunary statistically convergent to $A$, then $\left\{A_{k}\right\}$ is Wijsman quasi-almost lacunary statistically convergent to A.

Proof. Suppose that the sequence $\left\{A_{k}\right\}$ is Wijsman almost lacunary statistically convergent to $A$. Then, for every $\varepsilon, \delta>0$ and for each $x \in X$ there exists an integer $r_{0}>0$ such that for all $r>r_{0}$

$$
\frac{1}{h_{r}}\left|\left\{k \in I_{r}:\left|d_{x}\left(A_{k+i}\right)-d_{x}(A)\right| \geq \varepsilon\right\}\right|<\delta,
$$

uniformly in $i$. If $i$ is taken as $i=n r$, then we have

$$
\frac{1}{h_{r}}\left|\left\{k \in I_{r}:\left|d_{x}\left(A_{k+n r}\right)-d_{x}(A)\right| \geq \varepsilon\right\}\right|<\delta
$$

uniformly in $n$. Since $\delta>0$ is an arbitrary, we have

$$
\lim _{r \rightarrow \infty} \frac{1}{h_{r}}\left|\left\{k \in I_{r}:\left|d_{x}\left(A_{k+n r}\right)-d_{x}(A)\right| \geq \varepsilon\right\}\right|=0,
$$

uniformly in $n$ which means that $A_{k} \stackrel{(W Q S)_{\theta}}{\longrightarrow} A$.

Theorem 2.4. For any lacunary sequence $\theta=\left\{k_{r}\right\}$; if $\liminf _{r} q_{r}>1$, then $\{W Q S\} \subset\left\{W Q S_{\theta}\right\}$.

Proof. Suppose that $\liminf _{r} q_{r}>1$. Then for each $r \geq 1$, there is a number $\delta \geq 0$ such that $q_{r} \geq 1+\delta$. Since $q_{r} \geq 1+\delta$ and $h_{r}=k_{r}-k_{r-1}$, we have

$$
\frac{h_{r}}{k_{r}} \geq \frac{\delta}{1+\delta}
$$

Assume that $A_{k} \stackrel{W Q S}{\longrightarrow} A$. For each $x \in X$, we can write

$$
\begin{aligned}
\frac{1}{k_{r}}\left|\left\{k \leq k_{r}:\left|d_{x}\left(A_{k+n k_{r}}\right)-d_{x}(A)\right| \geq \varepsilon\right\}\right| & \geq \frac{1}{k_{r}}\left|\left\{k \in I_{r}:\left|d_{x}\left(A_{k+n k_{r}}\right)-d_{x}(A)\right| \geq \varepsilon\right\}\right| \\
& =\frac{h_{r}}{k_{r}}\left(\frac{1}{h_{r}}\left|\left\{k \in I_{r}:\left|d_{x}\left(A_{k+n k_{r}}\right)-d_{x}(A)\right| \geq \varepsilon\right\}\right|\right) \\
& \geq \frac{\delta}{1+\delta}\left(\frac{1}{h_{r}}\left|\left\{k \in I_{r}:\left|d_{x}\left(A_{k+n k_{r}}\right)-d_{x}(A)\right| \geq \varepsilon\right\}\right|\right)
\end{aligned}
$$

that is,

$$
\frac{1}{k_{r}}\left|\left\{k \leq k_{r}:\left|d_{x}\left(A_{k+n k_{r}}\right)-d_{x}(A)\right| \geq \varepsilon\right\}\right| \geq \frac{\delta}{1+\delta}\left(\frac{1}{h_{r}}\left|\left\{k \in I_{r}:\left|d_{x}\left(A_{k+n k_{r}}\right)-d_{x}(A)\right| \geq \varepsilon\right\}\right|\right)
$$


uniformly in $n$. If the limit is taken for the above inequality; since $A_{k} \stackrel{W Q S}{\longrightarrow} A$, we have

$$
0 \geq \frac{\delta}{1+\delta} \cdot \lim _{r \rightarrow \infty} \frac{1}{h_{r}}\left|\left\{k \in I_{r}:\left|d_{x}\left(A_{k+n k_{r}}\right)-d_{x}(A)\right| \geq \varepsilon\right\}\right| .
$$

By the definition of lacunary sequence, we can write $r$ instead of $k_{r}$. Hence, for each $x \in X$ we have

$$
\lim _{r \rightarrow \infty} \frac{1}{h_{r}}\left|\left\{k \in I_{r}:\left|d_{x}\left(A_{k+n r}\right)-d_{x}(A)\right| \geq \varepsilon\right\}\right|=0,
$$

uniformly in $n$, that is $A_{k} \stackrel{(W Q S)_{\theta}}{\longrightarrow} A$.

Definition 2.3. Let $\theta=\left\{k_{r}\right\}$ be a lacunary sequence. A sequence $\left\{A_{k}\right\} \in L_{\infty}$ is Wijsman quasi-strongly almost lacunary convergent to $A$ if for each $x \in X$,

$$
\frac{1}{h_{r}} \sum_{k \in I_{r}}\left|d_{x}\left(A_{k+n r}\right)-d_{x}(A)\right| \longrightarrow 0 \quad(\text { as } r \rightarrow \infty) ;
$$

uniformly in $n$. In this case, we will write $[W Q F]_{\theta}-\lim A_{k}=A$ or $A_{k} \stackrel{[W Q F]_{\theta}}{\longrightarrow} A$.

Theorem 2.5. For any lacunary sequence $\theta=\left\{k_{r}\right\}$; if $\liminf _{r} q_{r}>1$, then

$$
A_{k} \stackrel{[W Q F]}{\longrightarrow} A \Rightarrow A_{k} \stackrel{[W Q F]_{\theta}}{\longrightarrow} A .
$$

Proof. Let $\liminf { }_{r} q_{r}>1$. Then for each $r \geq 1$, there is a number $\delta \geq 0$ such that $q_{r} \geq 1+\delta$. Since $q_{r} \geq 1+\delta$ and $h_{r}=k_{r}-k_{r-1}$, we have

$$
\frac{k_{r}}{h_{r}} \leq \frac{1+\delta}{\delta} \quad \text { and } \quad \frac{k_{r-1}}{h_{r}} \leq \frac{1}{\delta}
$$

Assume that $A_{k} \stackrel{[W Q F]}{\longrightarrow} A$. For each $x \in X$, we can write

$$
\begin{aligned}
\frac{1}{h_{r}} \sum_{k \in I_{r}}\left|d_{x}\left(A_{k+n r}\right)-d_{x}(A)\right|= & \frac{1}{h_{r}} \sum_{i=1}^{k_{r}}\left|d_{x}\left(A_{i+n r}\right)-d_{x}(A)\right|-\frac{1}{h_{r}} \sum_{i=1}^{k_{r-1}}\left|d_{x}\left(A_{i+n r}\right)-d_{x}(A)\right| \\
= & \frac{k_{r}}{h_{r}}\left(\frac{1}{k_{r}} \sum_{i=1}^{k_{r}}\left|d_{x}\left(A_{i+n r}\right)-d_{x}(A)\right|\right) \\
& -\frac{k_{r-1}}{h_{r}}\left(\frac{1}{k_{r-1}} \sum_{i=1}^{k_{r-1}}\left|d_{x}\left(A_{i+n r}\right)-d_{x}(A)\right|\right) .
\end{aligned}
$$

Hence, for each $x \in X$ we have

$$
\begin{aligned}
\lim _{r \rightarrow \infty} \frac{1}{h_{r}} \sum_{k \in I_{r}}\left|d_{x}\left(A_{k+n r}\right)-d_{x}(A)\right|= & \lim _{r \rightarrow \infty} \frac{k_{r}}{h_{r}}\left(\frac{1}{k_{r}} \sum_{i=1}^{k_{r}}\left|d_{x}\left(A_{i+n r}\right)-d_{x}(A)\right|\right) \\
& -\lim _{r \rightarrow \infty} \frac{k_{r-1}}{h_{r}}\left(\frac{1}{k_{r-1}} \sum_{i=1}^{k_{r-1}}\left|d_{x}\left(A_{i+n r}\right)-d_{x}(A)\right|\right)
\end{aligned}
$$

uniformly in $n$. Since $A_{k} \stackrel{[W Q F]}{\longrightarrow} A$, for each $x \in X$ we have

$$
\frac{1}{k_{r}} \sum_{i=1}^{k_{r}}\left|d_{x}\left(A_{i+n r}\right)-d_{x}(A)\right| \rightarrow 0 \quad \text { and } \quad \frac{1}{k_{r-1}} \sum_{i=1}^{k_{r-1}}\left|d_{x}\left(A_{i+n r}\right)-d_{x}(A)\right| \rightarrow 0
$$


uniformly in $n$. By using the Inequalities (2.2) and the Status (2.3), we handle

$$
\lim _{r \rightarrow \infty} \frac{1}{h_{r}} \sum_{k \in I_{r}}\left|d_{x}\left(A_{k+n r}\right)-d_{x}(A)\right|=0
$$

The proof of theorem is completed.

Definition 2.4. Let $\theta=\left\{k_{r}\right\}$ be a lacunary sequence. A sequence $\left\{A_{k}\right\} \in L_{\infty}$ is Wijsman quasi q-strongly almost lacunary convergent to $A$ if for each $x \in X$ and $0<q<\infty$,

$$
\frac{1}{h_{r}} \sum_{k \in I_{r}}\left|d_{x}\left(A_{k+n r}\right)-d_{x}(A)\right|^{q} \longrightarrow 0 \quad(\text { as } r \rightarrow \infty)
$$

uniformly in $n$. In this case, we will write $[W Q F]_{\theta}^{q}-\lim A_{k}=A$ or $A_{k} \stackrel{[W Q F]_{\theta}^{q}}{\longrightarrow} A$.

Theorem 2.6. Let $0<q<\infty$. Then, we have following assertions:

i. If a sequence $\left\{A_{k}\right\}$ is Wijsman quasi q-strongly almost lacunary convergent to $A$, then the sequence $\left\{A_{k}\right\}$ is Wijsman quasi-almost lacunary statistically convergent to $A$.

ii. If a sequence $\left\{A_{k}\right\} \in L_{\infty}$ and Wijsman quasi-almost lacunary statistically convergent to $A$, then the sequence $\left\{A_{k}\right\}$ is Wijsman quasi q-strongly almost lacunary convergent to A.

Proof. (i) Let $\varepsilon>0$ be given. Then, for each $x \in X$ following inequality is proved

$$
\sum_{k \in I_{r}}\left|d_{x}\left(A_{k+n r}\right)-d_{x}(A)\right|^{q} \geq \varepsilon^{q}\left|\left\{k \in I_{r}:\left|d_{x}\left(A_{k+n r}\right)-d_{x}(A)\right| \geq \varepsilon\right\}\right|,
$$

uniformly in $n$. Since the sequence $\left\{A_{k}\right\}$ is Wijsman quasi $q$-strongly almost lacunary convergent to $A$; if the both side of Inequality (2.5) are multipled by $\frac{1}{h_{r}}$ and after that the limit is taken for $r \rightarrow \infty$, then we have

$$
\begin{aligned}
\lim _{r \rightarrow \infty} \frac{1}{h_{r}} \sum_{k \in I_{r}}\left|d_{x}\left(A_{k+n r}\right)-d_{x}(A)\right|^{q} & \geq \varepsilon^{q} \lim _{r \rightarrow \infty} \frac{1}{h_{r}}\left|\left\{k \in I_{r}:\left|d_{x}\left(A_{k+n r}\right)-d_{x}(A)\right| \geq \varepsilon\right\}\right| \\
0 & \geq \varepsilon^{q} \lim _{r \rightarrow \infty} \frac{1}{h_{r}}\left|\left\{k \in I_{r}:\left|d_{x}\left(A_{k+n r}\right)-d_{x}(A)\right| \geq \varepsilon\right\}\right| .
\end{aligned}
$$

Hence, we handle

$$
\lim _{r \rightarrow \infty} \frac{1}{h_{r}}\left|\left\{k \in I_{r}:\left|d_{x}\left(A_{k+n r}\right)-d_{x}(A)\right| \geq \varepsilon\right\}\right|=0,
$$

uniformly in $n$. That is $A_{k} \stackrel{(W Q S)_{\theta}}{\longrightarrow} A$.

(ii) Since $\left\{A_{k}\right\}$ is bounded, we can write

$$
\sup _{k}\left\{d_{x}\left(A_{k}\right)\right\}+d_{x}(A)=M, \quad(0<M<\infty),
$$

for each $x \in X$. 
If $\left\{A_{k}\right\}$ is Wijsman quasi-almost lacunary statistically convergent to $A$, then for a given $\varepsilon>0$ a number $N_{\varepsilon} \in \mathbb{N}$ can be chosen such that for all $r>N_{\varepsilon}$ and each $x \in X$

$$
\frac{1}{h_{r}}\left|\left\{k \in I_{r}:\left|d_{x}\left(A_{k+n r}\right)-d_{x}(A)\right| \geq\left(\frac{\varepsilon}{2}\right)^{1 / q}\right\}\right|<\frac{\varepsilon}{2 M^{q}}
$$

uniformly in $n$. Let take the set

$$
L_{p}=\left\{k \leq p:\left|d_{x}\left(A_{k+n r}\right)-d_{x}(A)\right| \geq\left(\frac{\varepsilon}{2}\right)^{1 / q}\right\} .
$$

Thus, for each $x \in X$ we have

$$
\begin{aligned}
\frac{1}{h_{r}} \sum_{k \in I_{r}}\left|d_{x}\left(A_{k+n r}\right)-d_{x}(A)\right|^{q} & =\frac{1}{h_{r}}\left(\sum_{\substack{k \in I_{r} \\
k \in L_{p}}}\left|d_{x}\left(A_{k+n r}\right)-d_{x}(A)\right|^{q}+\sum_{\substack{k \in I_{r} \\
k \notin L_{p}}}\left|d_{x}\left(A_{k+n r}\right)-d_{x}(A)\right|^{q}\right) \\
& <\frac{1}{h_{r}} h_{r} \frac{\varepsilon}{2 M^{q}} M^{q}+\frac{1}{h_{r}} h_{r} \frac{\varepsilon}{2} \\
& =\frac{\varepsilon}{2}+\frac{\varepsilon}{2}=\varepsilon
\end{aligned}
$$

uniformly in $n$. So, the proof is completed.

Theorem 2.7. If the sequence $\left\{A_{k}\right\}$ is Wijsman quasi q-strongly almost lacunary convergence to A, then $\left\{A_{k}\right\}$ is Wijsman q-strongly lacunary summable to $A$.

Proof. Suppose that the sequence $\left\{A_{k}\right\} \in L_{\infty}$ is Wijsman quasi $q$-strongly almost lacunary convergent to $A$. Then, Equation (2.4) is true which for $n=0$ implies for every $\varepsilon>0$ and each $x \in X$,

$$
\frac{1}{h_{r}} \sum_{k \in I_{r}}\left|d_{x}\left(A_{k}\right)-d_{x}(A)\right|^{q} \longrightarrow 0 \quad(\text { as } r \rightarrow \infty) ;
$$

so, $\left\{A_{k}\right\}$ is Wijsman $q$-strongly lacunary summable to $A$.

Theorem 2.8. If a sequence $\left\{A_{k}\right\}$ is Wijsman quasi q-strongly almost lacunary convergence to $A$, then the sequence $\left\{A_{k}\right\}$ is Wijsman lacunary statistically convergent to $A$.

Proof. Assume that the sequence $\left\{A_{k}\right\}$ is Wijsman quasi $q$-strongly almost lacunary convergence to $A$. Then, by Theorem 2.7 , the sequence $\left\{A_{k}\right\}$ is Wijsman $q$-strongly lacunary summable to $A$. For each $x \in X$ and every $\varepsilon>0$, we can write

$$
\sum_{k \in I_{r}}\left|d_{x}\left(A_{k}\right)-d_{x}(A)\right|^{q} \geq \varepsilon^{q}\left|\left\{k \in I_{r}:\left|d_{x}\left(A_{k}\right)-d_{x}(A)\right| \geq \varepsilon\right\}\right| .
$$

Since the sequence $\left\{A_{k}\right\}$ is Wijsman $q$-strongly lacunary summable to $A$; if the both side of Inequality (2.6) are multipled by $\frac{1}{h_{r}}$ and after that the limit is taken for $r \rightarrow \infty$, left side of the Inequality (2.6) is equal to 0. Hence, we handle

$$
\lim _{r \rightarrow \infty} \frac{1}{h_{r}}\left|\left\{k \in I_{r}:\left|d_{x}\left(A_{k}\right)-d_{x}(A)\right| \geq \varepsilon\right\}\right|=0 .
$$


The proof of theorem is completed.

\section{REFERENCES}

[1] A. R. Freedman, J. J. Sember and M. Raphael, Some Cesàro-type summability spaces, Proc. London Math. Soc. 37 (3) (1978), 508-520.

[2] D. Hajduković, Almost convergence of vector sequences, Mat. Vesnik 12 (27) (1975), 245-249.

[3] D. Hajduković, Quasi-almost convergence in a normed space, Univ. Beograd. Publ. Elektrotehn. Fak. Ser. Mat. 13 (2002), $36-41$.

[4] E. Gülle and U. Ulusu, Quasi-almost convergence of sequences of sets, J. Inequal. Spec. Funct. (in press).

[5] F. Nuray, Quasi-invariant convergence in a normed space, Annals of the University of Craiova, Mathematics and Computer Science Series 41 (1) (2014), 1-5.

[6] F. Nuray and B.E. Rhoades, Statistical convergence of sequences of sets, Fasc. Math. 49 (2012), 87-99.

[7] G. Beer, On convergence of closed sets in a metric space and distance functions, Bull. Aust. Math. Soc. 31 (1985), 421-432.

[8] G. Beer, Wijsman convergence: A survey, Set-Valued Anal. 2 (1994), 77-94.

[9] G. G. Lorentz, A contribution to the theory of divergent sequences, Acta Math. 80 (1948), 167-190.

[10] H. Fast, Sur la convergence statistique, Colloq. Math. 2 (1951), 241-244.

[11] I. J. Maddox, A new type of convergence, Math. Proc. Cambridge Philos. Soc. 83 (1978), 61-64.

[12] J. A. Fridy, On statistical convergence, Analysis 5 (1985), 301-313.

[13] J. A. Fridy and C. Orhan, Lacunary Statistical Convergence, Pac. J. Math. 160 (1) (1993), 43-53.

[14] M. Baronti and P. Papini, Convergence of sequences of sets, In: Methods of functional analysis in approximation theory, ISNM 76, Birkhauser-Verlag, Basel 1986.

[15] R. A. Wijsman, Convergence of sequences of convex sets, cones and functions, Bull. Amer. Math. Soc. 70 (1964), $186-188$.

[16] R. A. Wijsman, Convergence of sequences of convex sets, cones and functions II, Trans. Amer. Math. Soc. 123 (1) (1966), $32-45$.

[17] T. Šalát, On statistically convergent sequences of real numbers, Math. Slovaca 30 (1980), 139-150.

[18] U. Ulusu, On almost asymptotically lacunary statistical equivalence of sequences of sets, Electr. J. Math. Anal. Appl. 2 (2) (2014), 56-66.

[19] U. Ulusu, Lacunary statistical convergence of sequences of sets, Ph.D. Thesis, Afyon Kocatepe University, Institue of Science and Technology (2013).

[20] U. Ulusu and F. Nuray, On strongly lacunary summability of sequences of sets, J. Appl. Math. Bioinf. 3 (3) (2013), $75-88$.

[21] U. Ulusu and F. Nuray, Lacunary Statistical Convergence of Sequences of Sets, Progr. Appl. Math. 4 (2) (2012), $99-109$. 